\title{
Skeletal muscle wasting and long-term prognosis in patients undergoing rectal cancer surgery without neoadjuvant therapy
}

\author{
Alessandro Giani ${ }^{1,2}$, Simone Famularo ${ }^{1,2}$, Alessandro Fogliati ${ }^{1,2}$, Luca Riva ${ }^{3}$, Nicolò Tamini ${ }^{2}$, Davide Ippolito ${ }^{3}$, \\ Luca Nespoli ${ }^{1,2}$, Marco Braga ${ }^{1,2}$ and Luca Gianotti ${ }^{1,2^{*}}$
}

\begin{abstract}
Background: Derangement of body composition has been associated with dismal long-term survival in several gastrointestinal cancers including rectal tumors treated with neoadjuvant therapies. The role of specific preoperative anthropometric indexes on the oncologic outcomes of patients undergoing upfront surgery for rectal cancer has not been investigated.

The aim of the study is to evaluate the association of body composition and overall survival in this specific cohort.

Methods: Lumbar computed tomography images, obtained within the 30 days previous to surgery, between January 2009 and December 2016, were used to calculate population-specific thresholds of muscle mass (sarcopenia), subcutaneous and visceral adiposity, visceral obesity, sarcopenic obesity, and myosteatosis. These body composition variables were related with overall survival (OS), tumor-specific survival (TSS), and disease-free survival (DFS). OS, TSS, and DFS were evaluated by the Kaplan-Meier method. Cox regression analysis was used to identify independent predictors of mortality, tumor-specific mortality, and recurrence, and data were presented as hazard ratio (HR) and 95\% confidence interval (CI).
\end{abstract}

Results: During the study period, 411 patients underwent rectal resection for cancer, and among these, 129 were without neoadjuvant chemoradiation. The median follow-up was 96.7 months. At the end of the follow-up, 41 patients (31.8\%) had died; of these, 26 (20.1\%) died for tumor-related reasons, and 36 (27.1\%) experienced disease recurrence.

One-, three-, and five-year OS was $95.7 \%, 86.0 \%$, and $76.8 \%$ for non-sarcopenic patients versus $82.4 \%, 58.8 \%$, and $40.0 \%$ for sarcopenic ones respectively $(p<0.001)$. Kaplan-Meier survival curves comparing sarcopenic and non-sarcopenic patients showed a significant difference in terms of OS (log-rank $<0.0001$ ). Through multivariate Cox regression, overall mortality risk was associated only with sarcopenia (HR 1.96; 95\% CI 1.03-3.74; $p=0.041)$. Disease stage IV and III (HR 13.75; 95\% Cl 2.89-65.6; $p<0.001$ and HR 4.72; 95\% Cl 1.06-21.1; $p=0.043$, respectively) and sarcopenia (HR 2.62; $95 \% \mathrm{Cl} 1.22-5.6 ; p=0.013$ ) were independently associated with TSS. The other body composition indexes investigated showed no significant association with prognosis.

Conclusions: These results support the inclusion of body composition assessment for prognostic stratification of rectal cancer patients undergoing upfront resection.

\footnotetext{
*Correspondence: luca.gianotti@unimib.it

${ }^{2}$ Department of Surgery, San Gerardo Hospital, Monza, Italy

Full list of author information is available at the end of the article
}

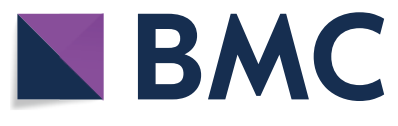

(c) The Author(s) 2022. Open Access This article is licensed under a Creative Commons Attribution 4.0 International License, which permits use, sharing, adaptation, distribution and reproduction in any medium or format, as long as you give appropriate credit to the original author(s) and the source, provide a link to the Creative Commons licence, and indicate if changes were made. The images or other third party material in this article are included in the article's Creative Commons licence, unless indicated otherwise in a credit line to the material. If material is not included in the article's Creative Commons licence and your intended use is not permitted by statutory regulation or exceeds the permitted use, you will need to obtain permission directly from the copyright holder. To view a copy of this licence, visit http://creativecommons.org/licenses/by/4.0/. The Creative Commons Public Domain Dedication waiver (http://creativeco mmons.org/publicdomain/zero/1.0/) applies to the data made available in this article, unless otherwise stated in a credit line to the data. 
Keywords: Sarcopenia, Body composition, Rectal cancer, Surgery, Long-term survival

\section{Introduction}

In recent years, the impact of anthropometry on outcome is a matter of extensive research in several fields of surgery $[1,2]$. Cancer-bearing patients are routinely staged before operation by way of computed tomography (CT) scan that also allows the estimation of quantitative and qualitative measurements of body composition $[3,4]$. The assessment of body structure through $\mathrm{CT}$ scan is used to stratify the risk of short-term postoperative morbidity and mortality in patients undergoing surgical procedures for malignant disease of the lung [5], liver [6], stomach [7], pancreas [8], and colorectum [9]. Likewise, specific body composition indexes seem to be associated with dismal long-term prognosis of patients suffering pancreatic, gastric, pulmonary, breast, biliary, and urinary tract tumors [5, 7, 10-13]. Recently, some studies have apprised the association of sarcopenia, visceral adiposity, and myosteatosis with worse overall and disease-free survival in patients undergoing colorectal surgery for cancer [14-17]. In those reports, colon and rectal cancers were evaluated as a single entity despite the fact that their perioperative management and treatment strategies are substantially different. Rectal surgery accounts for a substantially greater risk of postoperative complications and, in particular, higher rates of anastomotic leakage [18, 19] and surgical site infection [20] than those reported for colon resections. Moreover, rectal tumors appear to have a worse long-term prognosis than colonic ones [21-24]. Accordingly, it would be worthwhile to study more homogeneous patient populations. Neoadjuvant chemoradiation (NACR) should be the standard of care for stage II-III rectal cancer [25] given its advantages in terms of survival and recurrence. However, NACR is not indicated in the early and metastatic disease stages and its role is still debated in intermediate stage disease when a good quality mesorectal excision can be achieved [25-27]. Additionally, patients may not undergo NACR due to poor performance status or personal preference. Furthermore, neoadjuvant treatments may affect body composition [28] potentially resulting a confounding factor.

The aim of the present study is to evaluate the potential association of preoperative body composition indexes and long-term oncologic outcomes in a cohort of patients undergoing rectal resection for cancer without NACR.

\section{Materials and methods}

\section{Study overview}

A prospectively maintained and anonymized electronic dataset was queried for adult patients who had undergone curative surgery for rectal cancer between January 2009 and December 2016 at our institution. The exclusion criteria were the lack of a CT scan performed locally within 30 days previous to surgery and that they were receiving NACR. For data analysis, the oncologic follow-up was interrupted in May 2020. The local ethical committee review of the protocol deemed that formal approval was not required owing to the retrospective, observational, and anonymous nature of this study. The study protocol followed the ethical guidelines of the 1975 Declaration of Helsinki (revised in Brazil in 2013). Results are reported in accordance with Strengthening the Reporting of Observational Studies in Epidemiology (STROBE) [29].

\section{CT-derived body composition analysis}

The last CT scan performed before surgical resection was used for the analysis. A multiphasic multidetector CT scan was performed with two different CT scanners: The Brilliance $\mathrm{iCT}$ 256-slice or Brilliance 16-slice CT scanners (Philips Medical Systems, Eindhoven, Netherlands). An unenhanced scan was followed by a post-contrast triphasic acquisition (arterial, portal venous, and equilibrium phase), after the intravenous injection of 90 to $145 \mathrm{~mL}$ of non-ionic iodinated contrast medium (Xenetix 350; Guerbet, Aulnay, France) at a flow-rate of $3.5 \mathrm{~mL} / \mathrm{s}$ followed by the injection of $50 \mathrm{~mL}$ of saline solution. Images were reconstructed with the Filtered Back projection technique if acquired on Brilliance 16-slice CT or with the Hybrid-Iterative Reconstruction algorithm (iDose4) if acquired on Brilliance iCT. All the examinations were transferred to an image workstation (Intellispace portal 8.0; Philips Medical Systems) to evaluate, select, and save the image for analysis, in DICOM format. The analysis was performed with the open source image analysis software ImageJ (developed by the National Institutes of Health; available from http://rsbweb.nih. gov/ij/download.html), which gives comparable results of other soft-ware for body composition analysis, as previously described by van Vugt et al. [3].

Two dedicated radiologists (LR, DI), blinded to patient information, calculated total muscle area (TMA), which estimates the total muscle mass, total fat area (TFA), visceral fat area (VFA), subcutaneous fat area (SFA), and intramuscular fat area (IMFA) [4]. TMA, SFA and VFA were utilized to calculate the skeletal muscle index (SMI $\left.=\mathrm{TMA} / \mathrm{m}^{2}\right)$, subcutaneous adipose tissue index $(\mathrm{SATI}=$ $\mathrm{SFA} / \mathrm{m}^{2}$ ), and sarcopenic obesity (SO=VFA/TMA). The visceral to subcutaneous adipose tissue area ratio was 
calculated to explore the abdominal adipose tissue distribution $(\mathrm{VSR}=$ VFA/SFA). The grade of myosteatosis was determined through the intramuscular adipose tissue content (MS = IMFA/TMA). Body composition indexes were normalized for height in meters squared $[3,30]$ and expressed as $\mathrm{cm}^{2} / \mathrm{m}^{2}$. The first and the fourth quartile were estimated for each index according to sex [31]. Body composition indexes were presented as dichotomous variables. The first quartile of SMI was considered to be sarcopenia, while the last quartile was used for VATI, SATI, VSR, VFMAI, and IMFAR to quantify visceral adiposity, high subcutaneous adiposity, visceral obesity (VO), sarcopenic obesity (SO), and myosteatosis, respectively. Patients were considered obese when BMI $>30 \mathrm{~kg} / \mathrm{m}^{2}$ and underweight when $\mathrm{BMI}<20 \mathrm{~kg} / \mathrm{m}^{2}$ [30].

\section{Outcome variables and definitions}

Age, sex, the Charlson comorbidity index (CCI), and the American Society of Anesthesiology (ASA) score were collected during the first outpatient visit. The type of operation (anterior resection, Miles procedure or Hartmann procedure) and approach (open or laparoscopic) were the surgical parameters considered. Histology variables were evaluated and included infiltration of resection margins ( $\mathrm{R}$ status), pTNM, and disease stage. The Clavien-Dindo Classification was adopted to stratify postoperative morbidity and mortality [32]. The follow-up data included the date of death, the date of cancer relapse, and the site of relapse and were collected according to the latest outpatient visit. When oncologic follow-up was not available in the hospital registry, patients were reached by telephone interviews. Overall survival (OS) was defined as the time interval in months from surgery to death; if the patient was alive, data were censored at the last available visit. Tumor-specific survival (TSS) was defined as the time interval in months from surgery to cancerrelated death. Other specific causes of death were not recorded, but when occurred, patients were censored. Disease-free survival (DFS) was defined as the time interval in months from surgery to recurrence or death. In case of no recurrence, data were censored at the date of the last available follow-up. Patients with stage IV cancer were excluded from this latter analysis.

\section{Study endpoints}

The primary endpoint was to evaluate the possible association of different body composition indexes and OS in patients undergoing rectal resection for cancer who did not receive NACR. Secondary endpoints were the differences in terms of DFS and TSS among different anthropometric phenotypes.

\section{Statistics}

Data are expressed as a median and interquartile range (IQR) and number and relative percentage. Normal distribution of continuous variables was assessed using the Kolmogorov-Smirnov test. Continuous variables were analyzed using the Mann-Whitney test and categorical variables using the Fisher exact test or chi-square test as appropriate. OS, TSS, and DFS were evaluated using the Kaplan-Meier method. Stage IV patients were excluded from the DFS analysis. Comparison among groups was performed using the log-rank test. Cox regression analysis was used to identify independent predictors of mortality, tumor-specific mortality, and recurrence, and data was presented as hazard ratio (HR) and 95\% confidence interval (CI). Variables to be inserted in the model were selected by a step-forward approach with all the significant $(p<0.1)$ and clinically relevant variables examined by univariate Cox analysis. Median follow-up time was estimated with the reverse Kaplan-Meier method. All statistics were 2-tailed, and statistical significance was accepted when $p<0.05$. All statistical analyses were performed using R software (v 3.6.0).

\section{Results}

A total of 411 patients underwent a rectal resection for cancer during the study period. Among them, 129 patients did not receive NACR and thus were included in the analysis. The reasons they did not receive NACR were as follows: 13 patients were stage I, 30 were stage IV, 64 due to the upper tumor limit crossing over the peritoneal reflection, 13 due to comorbidities, and 9 due to refusal.

Baseline, surgical, and tumor characteristics are shown in Table 1 . Briefly, the median age of the cohort was 72 years (IQR 62-78), and 48 (37.2\%) were females. The median CCI was 5 (IQR 4-7), the median body mass index (BMI) 25.3 (IQR 23.4-27.8), and laparoscopic resection was performed for 62 patients (48.1\%). Body composition indexes, their relative ranges, and the cutoff values used for the present analysis are reported in Supplementary Table 1. The median follow-up was 96.7 months (95\% CI 61.6-119.5). At the end of the follow-up, overall, 41 patients (31.8\%) had died; of these, 26 (20.1\%) for tumor-related reasons. Thirty-five patients (27.1\%) experienced disease recurrence. Table 2 reports the Cox univariate analysis performed to identify factors predicting OS, TSS, and recurrence. The variables significantly associated with OS were age (HR 1.06, $p$ $<0.001$ ), CCI (HR 1.36, $p<0.001$ ), BMI (HR 0.88, $p=$ 0.006 ), SMI (HR 3.1, $p<0.001$ ), positive resection margins (HR 4.97, $p<0.001$ ), VATI (HR 0.36, $p=0.021$ ), and stage IV disease (HR 4.32, $p=0.003$ ). TSS resulted correlated with $\mathrm{CCI}(\mathrm{HR} 1.31, p=0.001$ ), BMI (HR 0.88, 
Table 1 Characteristics of the cohort

\begin{tabular}{|c|c|c|}
\hline \multicolumn{2}{|l|}{ Variables } & \multirow{2}{*}{$\begin{array}{l}\text { Included patients } N=129 \\
\text { Median (IQR)/N (\%) } \\
72(62-78)\end{array}$} \\
\hline Age & Years & \\
\hline Sex & Females & $48(37.2)$ \\
\hline BMI & & $25.3(23.4-27.8)$ \\
\hline $\mathrm{CCl}$ & & $5(4-7)$ \\
\hline \multirow[t]{3}{*}{ ASA score } & 1 & $4(3.1)$ \\
\hline & 2 & $66(51.2)$ \\
\hline & 3 & $59(45.7)$ \\
\hline \multirow[t]{3}{*}{ Type of surgery } & Anterior resection & $94(72.9)$ \\
\hline & Miles procedure & $21(16.3)$ \\
\hline & Hartmann procedure & $14(10.8)$ \\
\hline Laparoscopy & & $63(48.8)$ \\
\hline Overall morbidity & & $43(33.3)$ \\
\hline \multirow[t]{2}{*}{ Clavien-Dindo classification } & $<3$ & $27(20.9)$ \\
\hline & $\geq 3$ & $15(11.6)$ \\
\hline Redo surgery & & $8(6.2)$ \\
\hline Anastomotic leakage $^{a}$ & & $8 / 94(8.5)$ \\
\hline \multirow[t]{3}{*}{ Tumor location } & High & $64(49.6)$ \\
\hline & Middle & $41(31.8)$ \\
\hline & Low & $24(18.6)$ \\
\hline \multirow[t]{4}{*}{ TNM stage } & । & $30(23.3)$ \\
\hline & $\|$ & $33(25.6)$ \\
\hline & III & $41(31.8)$ \\
\hline & IV & $13(10.1)$ \\
\hline Positive resection margin & & $11(8.5)$ \\
\hline Adjuvant chemotherapy & & $61(47.3)$ \\
\hline
\end{tabular}

$B M I$ body mass index, CCI Charlson comorbidity index, ASA American Society of Anesthesiologists

${ }^{\text {a }}$ Calculated on the number of patients who underwent restorative surgery

$p=0.025)$, SMI (HR 2.99, $p=0.004)$, VATI (HR 0.18, $p=0.021$ ), positive resection margins (HR 7.96, $p<$ 0.001 ), TNM stage III (HR 5.53, $p=0.024$ ), TNM stage IV (HR 14.96, $p=0.001$ ), and adjuvant chemotherapy (HR 2.62, $p=0.028$ ). DFS was significantly associated with CCI (HR 1.23, $p=0.039)$ and BMI (HR 0.88, $p=$ 0.046). At the multivariate Cox regression, overall mortality risk was associated with sarcopenia (HR 1.96; 95\% CI 1.03-3.74; $p=0.041$ ) (Table 3). Disease stage IV and III (HR 13.75; 95\% CI 2.89-65.6; $p<0.001$ and HR 4.72; 95\% CI 1.06-21.1; $p=0.043$, respectively) and sarcopenia (HR 2.62; 95\% CI 1.22-5.6; $p=0.013$ ) were independently associated with TSS (Supplementary Fig. 1). One-, three-, and five-year OS was $95.7 \%, 86.0 \%$, and $76.8 \%$ for non-sarcopenic patients versus $82.4 \%, 58.8 \%$, and $40.0 \%$ for the sarcopenic group respectively $(p<0.001)$. TSS at 1,3 , and 5 years was $96.8 \%, 90.2 \%$, and $85.4 \%$ for nonsarcopenic patients versus $85.3 \%, 75.3 \%$, and $54.7 \%$ ( $p=$ 0.002 ) in the case of sarcopenia respectively. DFS rates at 1,3 , and 5 years were $89.4 \%, 80.3 \%$, and $70.6 \%$ for the non-sarcopenic group and $69.2 \%, 46.2 \%$, and $46.2 \%$ in the case of sarcopenia respectively $(p=0.004)$. The KaplanMeier survival curve for OS is depicted in Fig. 1 while TSS and DFS curves are shown in Supplementary Figs. 2 and 3 respectively.

\section{Discussion}

In this cohort study, regarding patients who underwent upfront rectal cancer surgery without NACR, the presence of preoperative sarcopenia assessed at $\mathrm{CT}$ scan was an independent prognostic factor for dismal longterm OS and TSS, along with other acknowledged variables such as the tumor stage. Several other studies have reported an association between preoperative skeletal muscle wasting and poor long-term survival in different oncological diseases [5-8, 33] and more specifically in colorectal cancer patients [15, 34-38]. However, all those trials used a combined analysis of colon and rectal cancer patients and this may have generated an ambiguous interpretation regarding the oncologic prognosis since 
Table 2 Values are hazard ration (HR) [95\% confidential intervals]. Stage IV patients were excluded from analysis of disease-free survival

\begin{tabular}{|c|c|c|c|}
\hline & Overall survival & Disease-free survival & Tumor-specific survival \\
\hline Age & $1.06(1.03-1.09, p<0.001)$ & $1.03(0.99-1.07, p=0.137)$ & $1.02(0.99-1.06, p=0.211)$ \\
\hline Sex, female & $0.79(0.43-1.44, p=0.444)$ & $0.67(0.27-1.64, p=0.376)$ & $0.69(0.32-1.52, p=0.360)$ \\
\hline $\mathrm{BMI}$ & $0.88(0.80-0.96, p=0.006)$ & $0.88(0.77-1.00, p=0.046)$ & $0.88(0.78-0.98, p=0.025)$ \\
\hline $\mathrm{CCl}$ & $1.36(1.20-1.53, p<0.001)$ & $1.23(1.01-1.50, p=0.039)$ & $1.31(1.12-1.53, p=0.001)$ \\
\hline \multicolumn{4}{|l|}{ ASA score } \\
\hline 2 vs 1 & $0.45(0.10-1.97, p=0.291)$ & $0.95(0.13-7.14, p=0.957)$ & $0.73(0.09-5.57, p=0.758)$ \\
\hline 3 vs 1 & $1.21(0.29-5.07, p=0.796)$ & $1.43(0.19-10.93, p=0.731)$ & $1.22(0.16-9.22, p=0.850)$ \\
\hline SMl, yes & $3.15(1.76-5.65, p<0.001)$ & $2.25(0.93-5.45, p=0.073)$ & $2.99(1.43-6.23, p=0.004)$ \\
\hline VO, yes & $1.02(0.54-1.94, p=0.947)$ & $1.97(0.84-4.61, p=0.118)$ & $0.85(0.36-1.98, p=0.703)$ \\
\hline IMFAR, yes & $1.28(0.69-2.40, p=0.435)$ & $0.71(0.26-1.92, p=0.495)$ & $0.78(0.32-1.92, p=0.590)$ \\
\hline SO, yes & $0.72(0.36-1.46, p=0.367)$ & $1.36(0.55-3.33, p=0.506)$ & $0.31(0.09-1.02, p=0.054)$ \\
\hline SATI, yes & $0.78(0.38-1.61, p=0.503)$ & $1.31(0.51-3.35, p=0.575)$ & $0.52(0.18-1.51, p=0.231)$ \\
\hline VATI, yes & $0.36(0.15-0.86, p=0.021)$ & $0.74(0.27-2.01, p=0.560)$ & $0.18(0.04-0.77, p=0.021)$ \\
\hline Laparoscopy, yes & $0.49(0.07-3.62, p=0.485)$ & $0.11(0.01-0.89, p=0.039)$ & $0.36(0.05-2.67, p=0.315)$ \\
\hline Severe complication, yes & $0.84(0.32-2.18, p=0.717)$ & $0.16(0.02-1.24, p=0.079)$ & $0.85(0.26-2.77, p=0.792)$ \\
\hline \multicolumn{4}{|l|}{ Tumor location } \\
\hline Middle vs high & $1.28(0.67-2.46, p=0.454)$ & $0.67(0.23-1.93, p=0.461)$ & $1.18(0.50-2.75, p=0.710)$ \\
\hline Low vs high & $1.37(0.63-2.98, p=0.434)$ & $1.64(0.57-4.72, p=0.362)$ & $1.46(0.56-3.85, p=0.441)$ \\
\hline \multicolumn{4}{|l|}{ TNM stage } \\
\hline || vs I & $1.63(0.68-3.94, p=0.277)$ & $1.23(0.33-4.58, p=0.759)$ & $3.00(0.61-14.87, p=0.179)$ \\
\hline|| $\mid$ vs $\mid$ & $1.68(0.72-3.93, p=0.232)$ & $2.88(0.94-8.84, p=0.064)$ & $5.53(1.25-24.51, p=0.024)$ \\
\hline$|V v s|$ & $4.32(1.65-11.27, p=0.003)$ & - & $14.96(3.16-70.84, p=0.001)$ \\
\hline \multicolumn{4}{|l|}{ Radicality } \\
\hline R1 vs R0 & $4.97(2.27-10.87, p<0.001)$ & - & $7.96(3.27-19.39, p<0.001)$ \\
\hline Adjuvant chemotherapy, yes & $1.19(0.66-2.17, p=0.563)$ & $2.36(0.92-6.04, p=0.072)$ & $2.62(1.11-6.19, p=0.028)$ \\
\hline
\end{tabular}

BMI Body mass index, CCI Charlson comorbidity index, ASA American Society of Anesthesiologists, SMI Skeletal muscle index, VATI Visceral adipose tissue index, SATI Subcutaneous adipose tissue index, VOVisceral obesity, SO Sarcopenic obesity

colonic tumors have a better survival than rectal cancers $[21-23,39]$. Only more recently have a few authors analyzed patients with rectal cancer alone [40, 41]. However, in these previous studies, all patients had received NACR. Since NACR may affect body composition variables [28], we decided to evaluate a specific cohort of patients

Table 3 Multivariate analysis of factors associated with overall mortality

\begin{tabular}{llll}
\hline & HR & $\mathbf{9 5 \% C l}$ & $\mathbf{p}$ \\
\hline Age (per year of increase) & 1.02 & $0.98-1.10$ & 0.398 \\
BMI (per point of increase) & 0.92 & $0.83-1.0$ & 0.107 \\
CCI (per point of increase) & 1.17 & $0.96-1.40$ & 0.124 \\
Sarcopenia (vs normal) & 1.96 & $1.03-3.7$ & 0.041 \\
Stage II (vs I) & 1.14 & $0.47-2.80$ & 0.775 \\
Stage III (vs I) & 1.29 & $0.54-3.10$ & 0.573 \\
Stage IV (vs I) & 2.46 & $0.82-7.40$ & 0.107 \\
VATI (low vs high) & 0.65 & $0.26-1.60$ & 0.347 \\
\hline
\end{tabular}

CI Confidential interval, BMI Body mass index, CCI Charlson comorbidity index, VATI Visceral adipose tissue index undergoing rectal resection without having received NACR. In this context, the present data may add value to the previous literature since this group of patients had not been previously investigated. However, even in this relatively small and selected population, preoperative muscle wasting was significantly associated with dismal long-term prognosis as in other studies [40, 41].

Other authors have evaluated the role of visceral obesity and found no significant difference in the DFS and OS between the group bearing this condition and controls $[16,40,42]$. Similar to these findings, we did not observe a significant association of adiposity-related indexes, namely visceral obesity, sarcopenic obesity and myosteatosis, and survival. In contrast, Brown et al. [17] recently demonstrated the role of visceral and subcutaneous adiposity in affecting mortality in a large cohort of North American colorectal cancer patients. Instead, Han et al. [43] showed in a Korean population, that sarcopenic obesity was the only anthropometric variable negatively associated with overall survival in a specific subgroup of non-metastatic rectal cancer patients. In another recent 


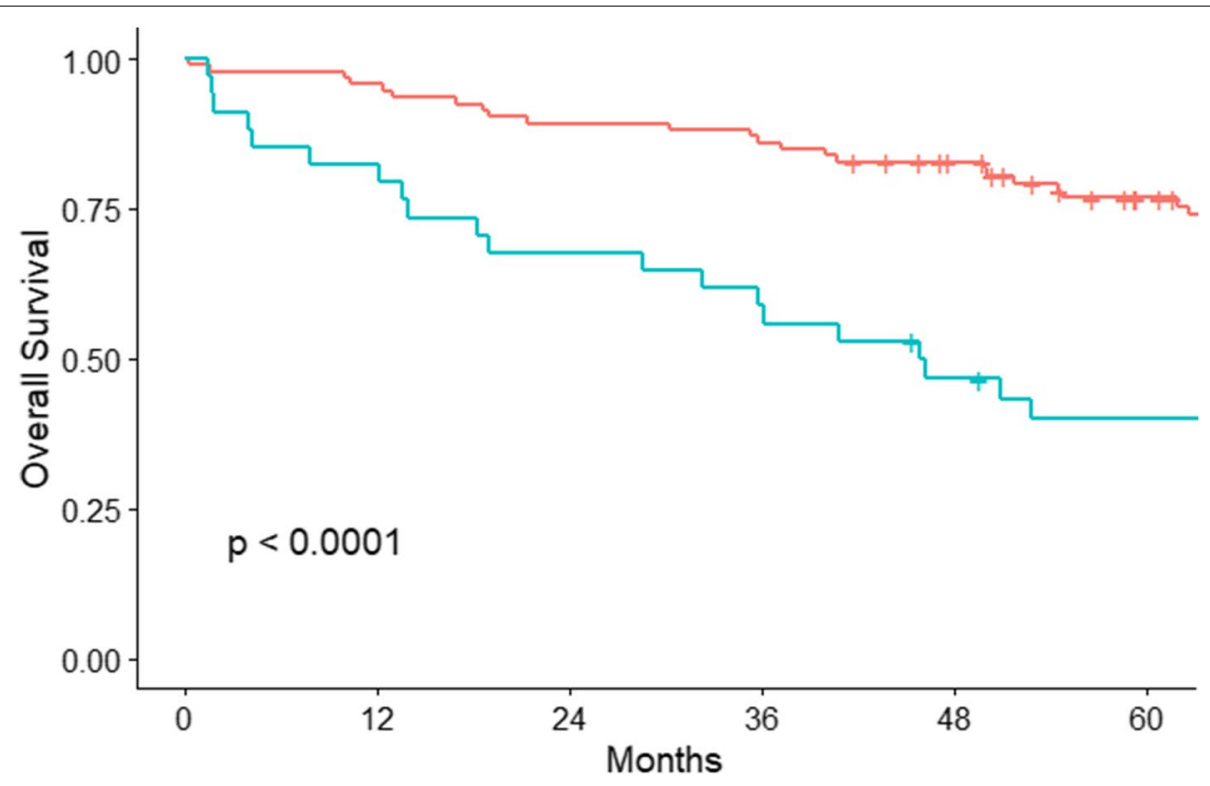

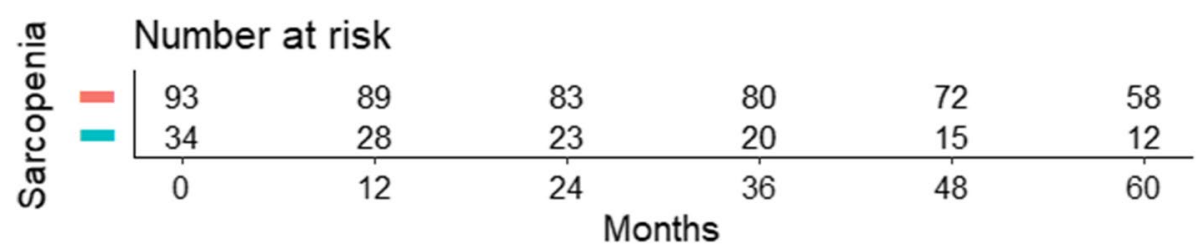

Sarcopenia $+\mathrm{No}+\mathrm{Yes}$

Fig. 1 Kaplan-Meier overall survival curves for sarcopenic and non sarcopenic rectal cancer patients

study, Horii et al. found a negative impact of high intramuscular adipose tissue content on short and long-term outcomes in a subgroup of patients with colorectal liver metastasis undergoing hepatectomy [44]. This inconsistency of results may be partially explained by the different ethnicity and the well-recognized differences in anthropometric phenotype among Mediterranean, North American, and Asian populations.

The exact mechanism of how changes in body composition affect survival of cancer patients remains speculative. Sarcopenia is a component of cancer cachexia and a hallmark of malnutrition, and thus, it may be considered an indirect sign of aggressive disease and subsequent poor prognosis [45]. Nutritional risk scores are widely used and have been repeatedly demonstrated to be predictors of all-cause mortality and cancer-specific death in colorectal cancer [46, 47]. However, some authors have underlined how the quantitative measurement of muscle wasting overtakes classic malnutrition metrics as a predictor of survival $[36,45]$, and the recently proposed GLIM criteria for malnutrition now include instrumental measurement of the muscle mass [48].
Different pathophysiologic pathways have been proposed to explain the association between muscle mass or other body compartments and long-term survival. A systemic inflammation status seems a key mechanism [49]. Chronic inflammation increases the risk of cancer and hampers patient response to treatments [50]. High preoperative inflammatory markers have been directly associated to an increase in morbidity and mortality in colorectal cancer patients undergoing surgery [51]. Adipose tissue and skeletal muscle are the largest organs of the body and their immunoregulatory role are well recognized, given the ability to release a wide range of bioactive mediators [52]. In particular, myokines, cytokines produced by myocytes, play a critical role in cancer prevention counterbalancing the harmful effects of proinflammatory adipokines $[52,53]$.

The qualitative and quantitative evaluation of body architecture based on CT scan has several advantages. It does not add risk to patients since this imaging tool is routinely used for staging purposes, and thanks to new software, the measurement of different body compartments can be easily gathered. Furthermore, accuracy and reproducibility $[3,40]$ are additional reasons to propose abdominal $\mathrm{CT}$ imaging 
as the reference technique for anthropometric evaluation. These characteristics, combined with the prognostic ability, suggest adding CT-derived body composition assessment to conventional evaluation tools to identify individuals who might benefit from early and tailored metabolic interventions. By moving from measurement to action, multimodal prehabilitation programs, combining physical exercise and personalized nutritional intervention, in candidates to colorectal operations, have been recently shown to increase lean body mass and reduce fat mass [54]. Whether these changes in body composition will affect long-term outcomes of surgical cancer patients need to be further explored.

There are some limitations in our study. First, it was a single-center retrospective study and results need to be validated by prospective observational trials. Second, we used sex-related percentiles of our population rather than validated cutoffs to define groups at risk. Yet, body composition may have profound regional and ethnic variations, and peculiar diseases may have different influences on anthropometric changes, emphasizing the risk of using universal thresholds. Moreover, CT scans were obtained at the time of diagnosis, and the trajectory and overtime changes were not investigated. Lastly, there was a relatively small number of patients who experienced recurrence and cancer-related death, with a potential generation of a type-II error.

\section{Conclusions}

In this cohort study of patients who underwent rectal cancer surgery without NACR, the presence of preoperative sarcopenia assessed at CT scan was an independent prognostic factor for dismal long-term survival. The present results support the inclusion of body composition assessment for prognostic stratification also for this cohort of patients.

\section{Supplementary Information}

The online version contains supplementary material available at https://doi. org/10.1186/s12957-021-02460-7.

Additional file 1: Supplementary Table 1 Descriptive analysis of the anthropometric measures and body composition indexes. Legend: Numbers are medians (percentile). BMI: Body mass index; SMI: Skeletal muscle index; VATI: Visceral adipose tissue index; SATI: Subcutaneous adipose tissue index; VFMAI:Visceral fat-muscle area index; VSR: Visceral to subcutaneous adipose tissue area ratio; IMFAR: Infra-muscular fat area ratio.

Additional file 2: Supplementary Fig. 1 Multivariate analysis of factors associated to cancer-related death in rectal cancer patients undergoing upfront surgery. Quadrangles represent hazard risk. Horizontal bars represent 95\% confidential interval VATI: Visceral adipose tissue index.

Additional file 3: Supplementary Fig. 2 Kaplan-Meier tumor-specific survival curve for sarcopenic and non sarcopenic rectal cancer patients.

Additional file 4: Supplementary Fig. 3 Kaplan-Meier disease-free survival curve for sarcopenic and non sarcopenic rectal cancer patients.
Acknowledgements

Not applicable.

\section{Authors' contributions}

Conceptualization, A.G. and L.G.; methodology, A.G., S.F. and L.G..; radiological images analysis, L.R. and D.I.; data analysis, S.F.; data curation, A.G., N.T., and A.F.; writing: original draft preparation, A. G, A.F., and S.F.; writing: review and editing, A.G., A.F., N.T., L.N., and L.G.; supervision, M.B. All authors have read and agreed to the published version of the manuscript.

\section{Funding}

This research received no external funding.

\section{Availability of data and materials}

The datasets analyzed during the current study are available from the corresponding author on reasonable request.

\section{Declarations}

Ethics approval and consent to participate

The local ethical committee reviewed the protocol and deemed that formal approval was not required owing to the retrospective, observational, and anonymous nature of this study. The study protocol followed the ethical guidelines of the 1975 Declaration of Helsinki (revised in Brazil 2013). The results are reported according to Strengthening the Reporting of Observational Studies in Epidemiology (STROBE). Informed consent was not obtained given the retrospective nature of the study.

\section{Consent for publication}

Not applicable.

\section{Competing interests}

The authors declare that they have no competing interests.

\section{Author details}

${ }^{1}$ Present Address: School of Medicine and Surgery, University of MilanoBicocca, Monza, Italy. ${ }^{2}$ Department of Surgery, San Gerardo Hospital, Monza, Italy. ${ }^{3}$ Department of Radiology, San Gerardo Hospital Via Pergolesi 33, 20900 Monza, Italy.

Received: 12 September 2021 Accepted: 29 November 2021

Published online: 25 February 2022

\section{References}

1. Abbass T, Dolan RD, Laird BJ, McMillan DC. The relationship between imaging-based body composition analysis and the systemic inflammatory response in patients with cancer: a systematic review. Cancers (Basel). 2019;11(9):1304.

2. Pring ET, Malietzis G, Kennedy RH, Athanasiou T, Jenkins JT. Cancer cachexia and myopenia - update on management strategies and the direction of future research for optimizing body composition in cancer - a narrative review. Cancer Treat Rev. 2018;70:245-54.

3. van Vugt JL, Levolger S, Gharbharan A, Koek M, Niessen WJ, Burger JW, et al. A comparative study of software programmes for cross-sectional skeletal muscle and adipose tissue measurements on abdominal computed tomography scans of rectal cancer patients. J Cachexia Sarcopenia Muscle. 2017:8(2):285-97.

4. Shen W, Punyanitya M, Wang Z, Gallagher D, St-Onge MP, Albu J, et al. Total body skeletal muscle and adipose tissue volumes: estimation from a single abdominal cross-sectional image. J Appl Physiol (1985). 2004;97(6):2333-8.

5. Kawaguchi Y, Hanaoka J, Ohshio Y, Okamoto K, Kaku R, Hayashi K, et al. Sarcopenia predicts poor postoperative outcome in elderly patients with lung cancer. Gen Thorac Cardiovasc Surg. 2019;67(11):949-54.

6. Peng PD, van Vledder MG, Tsai S, de Jong MC, Makary M, Ng J, et al. Sarcopenia negatively impacts short-term outcomes in patients undergoing hepatic resection for colorectal liver metastasis. HPB (Oxford). 2011;13(7):439-46. 
7. Zhuang CL, Huang DD, Pang WY, Zhou CJ, Wang SL, Lou N, et al. Sarcopenia is an independent predictor of severe postoperative complications and long-term survival after radical gastrectomy for gastric cancer: analysis from a large-scale cohort. Medicine (Baltimore). 2016;95(13):e3164.

8. Pecorelli N, Capretti G, Sandini M, Damascelli A, Cristel G, De Cobelli F, et al. Impact of sarcopenic obesity on failure to rescue from major complications following pancreaticoduodenectomy for cancer: results from a multicenter study. Ann Surg Oncol. 2018;25(1):308-17.

9. Giani A, Famularo S, Riva L, Tamini N, Ippolito D, Nespoli L, et al. Association between specific presurgical anthropometric indexes and morbidity in patients undergoing rectal cancer resection. Nutrition. 2020;75-76:110779.

10. Rier HN, Jager A, Sleijfer S, van Rosmalen J, Kock MCJM, Levin MD. Low muscle attenuation is a prognostic factor for survival in metastatic breast cancer patients treated with first line palliative chemotherapy. Breast. 2017:31:9-15.

11. van Dijk DP, Bakens MJ, Coolsen MM, Rensen SS, van Dam RM, Bours MJ, et al. Low skeletal muscle radiation attenuation and visceral adiposity are associated with overall survival and surgical site infections in patients with pancreatic cancer. J Cachexia Sarcopenia Muscle. 2017;8(2):317-26.

12. Chakedis J, Spolverato G, Beal EW, Woelfel I, Bagante F, Merath K, et al. Pre-operative sarcopenia identifies patients at risk for poor survival after resection of biliary tract cancers. J Gastrointest Surg. 2018;22(10):1697-708.

13. Mayr R, Gierth M, Zeman F, Reiffen M, Seeger P, Wezel F, et al. Sarcopenia as a comorbidity-independent predictor of survival following radical cystectomy for bladder cancer. J Cachexia Sarcopenia Muscle. 2018;9(3):505-13.

14. Malietzis G, Currie AC, Athanasiou T, Johns N, Anyamene N, Glynne-Jones $\mathrm{R}$, et al. Influence of body composition profile on outcomes following colorectal cancer surgery. Br J Surg. 2016;103(5):572-80.

15. Hopkins JJ, Reif RL, Bigam DL, Baracos VE, Eurich DT, Sawyer MB. The impact of muscle and adipose tissue on long-term survival in patients with stage I to III colorectal cancer. Dis Colon Rectum. 2019;62(5):549-60.

16. Park SE, Hwang IG, Choi CH, Kang H, Kim BG, Park BK, et al. Sarcopenia is poor prognostic factor in older patients with locally advanced rectal cancer who received preoperative or postoperative chemoradiotherapy. Medicine (Baltimore). 2018;97(48):e13363.

17. Brown JC, Caan BJ, Prado CM, Cespedes Feliciano EM, Xiao J, Kroenke CH, et al. The association of abdominal adiposity with mortality in patients with stage I-III colorectal cancer. J Natl Cancer Inst. 2020;1 12(4):377-83.

18. Leijssen LGJ, Dinaux AM, Kunitake H, Bordeianou LG, Berger DL. The impact of postoperative morbidity on survival in patients with metastatic colon and rectal cancer. J Surg Oncol. 2019;120(3):460-72.

19. Alves A, Panis Y, Mathieu P, Mantion G, Kwiatkowski F, Slim K. Association Française de Chirurgie. Postoperative mortality and morbidity in French patients undergoing colorectal surgery: results of a prospective multicenter study. Arch Surg. 2005;140(3):278-83 discussion 284

20. Konishi T, Watanabe T, Kishimoto J, Nagawa H. Elective colon and rectal surgery differ in risk factors for wound infection: results of prospective surveillance. Ann Surg. 2006;244(5):758-63.

21. McDermott FT, Hughes ES, Pihl E, Milne BJ, Price AB. Comparative results of surgical management of single carcinomas of the colon and rectum: a series of 1939 patients managed by one surgeon. $\mathrm{Br} J$ Surg. 1981;68(12):850-5.

22. Lee YC, Lee YL, Chuang JP, Lee JC. Differences in survival between colon and rectal cancer from SEER data. PLoS One. 2013;8(11):e78709.

23. Li M, Li JY, Zhao AL, Gu J. Colorectal cancer or colon and rectal cancer? Clinicopathological comparison between colonic and rectal carcinomas. Oncology. 2007;73(1-2):52-7.

24. Iqbal A, George TJ. Randomized clinical trials in colon and rectal cancer. Surg Oncol Clin N Am. 2017;26(4):689-704.

25. Glynne-Jones R, Wyrwicz L, Tiret E, Brown G, Rödel C, Cervantes A, et al. Rectal cancer: ESMO Clinical Practice Guidelines for diagnosis, treatment and follow-up. Ann Oncol. 2017;28(suppl_4):iv22-40.

26. Li Y, Wang J, Ma X, Tan L, Yan Y, Xue C, et al. A review of neoadjuvant chemoradiotherapy for locally advanced rectal cancer. Int J Biol Sci. 2016;12(8):1022-31.

27. MERCURY Study Group. Diagnostic accuracy of preoperative magnetic resonance imaging in predicting curative resection of rectal cancer: prospective observational study. BMJ. 2006;333(7572):779.
28. Davis MP, Panikkar R. Sarcopenia associated with chemotherapy and targeted agents for cancer therapy. Ann Palliat Med. 2019;8(1):86-101.

29. von Elm E, Altman DG, Egger M, Pocock SJ, Gøtzsche PC, Vandenbroucke $J P$, et al. The Strengthening the Reporting of Observational Studies in Epidemiology (STROBE) Statement: guidelines for reporting observational studies. Int J Surg. 2014;12(12):1495-9.

30. Prado CM, Lieffers JR, McCargar LU, Reiman T, Sawyer MB, Martin L, et al. Prevalence and clinical implications of sarcopenic obesity in patients with solid tumours of the respiratory and gastrointestinal tracts: a populationbased study. Lancet Oncol. 2008;9(7):629-35.

31. Miyamoto Y, Baba Y, Sakamoto Y, Ohuchi M, Tokunaga R, Kurashige J, et al. Sarcopenia is a negative prognostic factor after curative resection of colorectal cancer. Ann Surg Oncol. 2015;22(8):2663-8.

32. Dindo D, Demartines N, Clavien PA. Classification of surgical complications: a new proposal with evaluation in a cohort of 6336 patients and results of a survey. Ann Surg. 2004;240(2):205-13.

33. Järvinen T, Ilonen I, Kauppi J, Salo J, Räsänen J. Loss of skeletal muscle mass during neoadjuvant treatments correlates with worse prognosis in esophageal cancer: a retrospective cohort study. World I Surg Oncol. 2018;16(1):27.

34. Aro R, Mäkäräinen-Uhlbäck E, Ämmälä N, Rautio T, Ohtonen P, Saarnio $J$, et al. The impact of sarcopenia and myosteatosis on postoperative outcomes and 5-year survival in curatively operated colorectal cancer patients - a retrospective register study. Eur J Surg Oncol. 2020;46(9):1656-62.

35. Okugawa Y, Toiyama Y, Yamamoto A, Shigemori T, Yin C, Narumi A, et al. Clinical impact of muscle quantity and quality in colorectal cancer patients: a propensity score matching analysis. JPEN J Parenter Enteral Nutr. 2018;42(8):1322-33.

36. Vashi PG, Gorsuch K, Wan L, Hill D, Block C, Gupta D. Sarcopenia supersedes subjective global assessment as a predictor of survival in colorectal cancer. PLoS One. 2019;14(6):e0218761.

37. Shirdel M, Andersson F, Myte R, Axelsson J, Rutegård M, Blomqvist L, et al. Body composition measured by computed tomography is associated with colorectal cancer survival, also in early-stage disease. Acta Oncol. 2020;59(7):799-808.

38. Lee CS, Won DD, Oh SN, Lee YS, Lee IK, Kim IH, et al. Prognostic role of pre-sarcopenia and body composition with long-term outcomes in obstructive colorectal cancer: a retrospective cohort study. World J Surg Oncol. 2020;18(1):230

39. van den Berg I, Coebergh van den Braak RRJ, van Vugt JLA, ljzermans JNM, Buettner S. Actual survival after resection of primary colorectal cancer: results from a prospective multicenter study. World J Surg Oncol. 2021;19(1):96.

40. Takeda Y, Akiyoshi T, Matsueda K, Fukuoka H, Ogura A, Miki H, et al. Skeletal muscle loss is an independent negative prognostic factor in patients with advanced lower rectal cancer treated with neoadjuvant chemoradiotherapy. PLoS One. 2018;13(4):e0195406.

41. Levolger S, van Vledder MG, Alberda WJ, Verhoef C, de Bruin RWF, IJzermans JNM, Burger JW. Muscle wasting and survival following preoperative chemoradiotherapy for locally advanced rectal carcinoma. Clin Nutr. 2018;37(5):1728-35.

42. Choi MH, Oh SN, Lee IK, Oh ST, Won DD. Sarcopenia is negatively associated with long-term outcomes in locally advanced rectal cancer. J Cachexia Sarcopenia Muscle. 2018;9(1):53-9.

43. Han JS, Ryu H, Park IJ, Kim KW, Shin Y, Kim SO, et al. Association of body composition with long-term survival in non-metastatic rectal cancer patients. Cancer Res Treat. 2020;52(2):563-72.

44. Horii N, Sawda Y, Kumamoto T, Tsuchiya N, Murakami T, Yabushita Y, et al. Impact of intramuscular adipose tissue content on short- and long-term outcomes of hepatectomy for colorectal liver metastasis: a retrospective analysis. World J Surg Oncol. 2020;18(1):68.

45. Hopkinson JB, Wright DN, McDonald JW, Corner JL. The prevalence of concern about weight loss and change in eating habits in people with advanced cancer. J Pain Symptom Manag. 2006;32(4):322-31.

46. Doleman B, Mills KT, Lim S, Zelhart MD, Gagliardi G. Body mass index and colorectal cancer prognosis: a systematic review and meta-analysis. Tech Coloproctol. 2016;20(8):517-35.

47. Luvián-Morales J, González-Trejo S, Carrillo JF, Herrera-Goepfert R, Aiello-Crocifoglio V, Gallardo-Rincón D, et al. Association of the prognostic nutritional index and overall survival in patients with colorectal 
cancer: A STROBE compliant retrospective cohort study. Cancer Med. 2019;8(7):3379-88.

48. Cederholm T, Jensen GL, Correia MITD, Gonzalez MC, Fukushima R, Higashiguchi T, et al. GLIM criteria for the diagnosis of malnutrition - a consensus report from the global clinical nutrition community. Clin Nutr. 2019;38(1):1-9.

49. Feliciano EMC, Kroenke CH, Meyerhardt JA, Prado CM, Bradshaw PT, Kwan $\mathrm{ML}$, et al. Association of systemic inflammation and sarcopenia with survival in nonmetastatic colorectal cancer: results from the C SCANS study. JAMA Oncol. 2017;3(12):e172319.

50. Coussens LM, Werb Z. Inflammation and cancer. Nature. 2002;420(6917):860-7.

51. Alsaif SH, Rogers AC, Pua P, Casey PT, Aherne GG, Brannigan AE, et al. Preoperative $\mathrm{C}$-reactive protein and other inflammatory markers as predictors of postoperative complications in patients with colorectal neoplasia. World J Surg Oncol. 2021;19(1):74.

52. Trayhurn P, Drevon CA, Eckel J. Secreted proteins from adipose tissue and skeletal muscle - adipokines, myokines and adipose/muscle cross-talk. Arch Physiol Biochem. 2011;117(2):47-56.

53. Miles L. The new WCRF/AICR report - food, nutrition, physical activity and the prevention of cancer: a global perspective. Nutr Bull. 2008;33:26-32.

54. Gillis C, Fenton TR, Sajobi TT, Minnella EM, Awasthi R, Loiselle SĖ, et al. Trimodal prehabilitation for colorectal surgery attenuates post-surgical losses in lean body mass: a pooled analysis of randomized controlled trials. Clin Nutr. 2019;38(3):1053-60.

\section{Publisher's Note}

Springer Nature remains neutral with regard to jurisdictional claims in published maps and institutional affiliations.

- fast, convenient online submission

- thorough peer review by experienced researchers in your field

- rapid publication on acceptance

- support for research data, including large and complex data types

- gold Open Access which fosters wider collaboration and increased citations

- maximum visibility for your research: over $100 \mathrm{M}$ website views per year

At BMC, research is always in progress.

Learn more biomedcentral.com/submissions 